\title{
THE PREVALENCE OF DYSPLASIA IN COLORECTAL SERRATED/HYPERPLASTIC POLYPS IN OMANI POPULATION
}

\author{
Asim Qureshi ${ }^{1}$, Nawras al Hadi ${ }^{2}$, Asem Shalaby ${ }^{1}$ \\ ${ }^{1}$ Department of Pathology, Sultan Qaboos University, Muscat Oman, ${ }^{2}$ Medical Student, College of Medicine Sultan \\ Qaboos University Oman, Oman
}

Received: 19 November 2018 / Accepted: 11 March 2019

\begin{abstract}
Purpose: Serrated/hyperplastic polyps (SPs) are characterised histologically by sawtooth architecture. Historically, these polyps were considered benign, without malignant potential and thus clinically unimportant. At present, the WHO defines serrated/hyperplastic lesions as heterogeneous group, which include hyperplastic polyps (HP), sessile serrated adenoma (SSA)/polyp and traditional serrated adenoma (TSA). These can have malignant potential. The aim of this study was to evaluate the prevalence of dysplastic changes in SP of colon and rectum of Omani population reported at a tertiary care centre.
\end{abstract}

Methods: The study was conducted in the pathology department of a tertiary care centre including endoscopic polypectomies from colon and rectum of Omani patients presenting to gastroenterology clinic reported between 2014 and 2016, and these were analysed retrospectively for dysplastic changes and their association with different clinical parameters.

Results: Dysplasia was seen in 32 of 146 cases (21.9\%) and 114 (78.1\%) were without dysplasia. Of these dysplastic, biopsies' only two cases (1.4\%) showed high-grade dysplasia and rest 30 (20.5\%) low-grade dysplasia. According to the type of polyp, dysplasia was found in TSA 8/21 cases (46.7\%) and SSA 9/17 cases (53.3\%). Association of dysplasia was more common in the age group of $>50$ (15.07\%) and male patients $(62.5 \%)$.

Conclusion: SPs are now common findings that a gastroenterologist faces in their daily practice. In our study of Omani population, the prevalence of dysplasia is associated with older age group, male gender, SSA/polyp and traditional serrated adenoma.

Key words: Dysplasia. colorectal, polyps

\section{Introduction}

Polyp is a histological term that describes any circumscribed growth that projects above the surrounding mucosa. The term polyp itself has no ominous clinical significance. Polyp can be neoplastic, inflammatory or hamartomatous. Only histological examination can reveal their true identity, we can appreciate their clinical behaviour. ${ }^{[1]}$

In general, intestinal polyps are classified as nonneoplastic or neoplastic. Most common neoplastic polyps are adenomas or adenomatous polyps, which arise due to dysplastic proliferation and have a potency to progress to

Correspondence: Dr. Asim Qureshi, Department of Pathology, Sultan Qaboos University, Muscat, Oman. Email asimqureshi32@hotmail.com cancer. The other types of polyps include hamartomatous, inflammatory and serrated/hyperplastic polyps (SPs). ${ }^{[1]}$

SPs are those characterised by sawtooth architecture from which it got its name. Historically, these polyps were considered benign, without malignant potential and thus clinically unimportant. ${ }^{[2]}$ At present, the WHO defines serrated/hyperplastic lesions as heterogeneous group, which include hyperplastic polyps (HPs), sessile serrated adenoma (SSA)/polyp and traditional serrated adenoma (TSA). ${ }^{[3]}$ These can have malignant potential. ${ }^{[3]}$

HPs are the most common type of SPs accounting for $>75 \%$ of all serrated polyps. ${ }^{[4]}$ Typically seen more in the left colon and, in particular, sigmoid and rectum, 
especially in the sixth and seven decades ${ }^{[5]}$ with no difference in occurrence between males and females. ${ }^{[6]}$ SSPs are distinguished from HPs by crypt distortion, anchor-shaped or boot-shaped crypts, shown in Figure 1. TSAs lined by hypereosinophilic or pencillate cells. ${ }^{[7]}$ They represent a hybrid of serrated and conventional adenomas [Figure 2].

It is postulated that sessile serrated polyp pathway starts with a mutation in BRAF oncogene; this mutation promotes cellular proliferation and downregulation of apoptosis pathway. ${ }^{[8]}$

The aim of this study was to evaluate the degree of dysplastic changes in SPs of colon and rectum of Omani population reported at a tertiary care centre in Oman.

\section{Materials and Methods}

This is a retrospective study. Ethical approval for this study was obtained.

Blocks and slides of tissue taken by colonoscopy of patients presenting to the gastroenterology clinic over a 3-year period were retrieved through hospital information system. These were reevaluated for dysplasia by two pathologists.

All polyps reported as hyperplastic/serrated over a period of 2014-2016 were included in the study regardless of their age or gender. Polyps from other sites in the gastrointestinal tract and non-serrated lesion including adenomatous polyps and inflammatory polyps regardless for their site were excluded from this study.

Clinical parameters which were taken into account were age and sex of the patient, and age and site of polyp. The patients were grouped into three age groups: $<18$ years representing paediatric population, $18-50$ years represent adult age group and patient $>50$ years old representing older population. The site of lesion was classified on the various parts of colon including cecum, ascending colon, transverse colon, descending colon, sigmoid colon and rectum. The lesions located in the hepatic flexures were regarded as ascending colon and those located in splenic flexure as descending colon. Finally, all the lesions located in cecum, ascending colon and transverse colon were regarded as proximal colon, while those located in

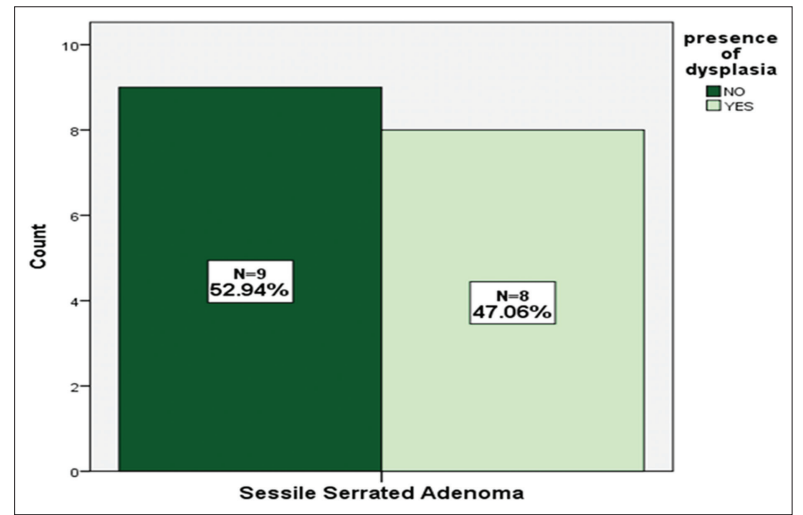

Figure 1: The prevalence of dysplasia in sessile serrated adenoma of this study

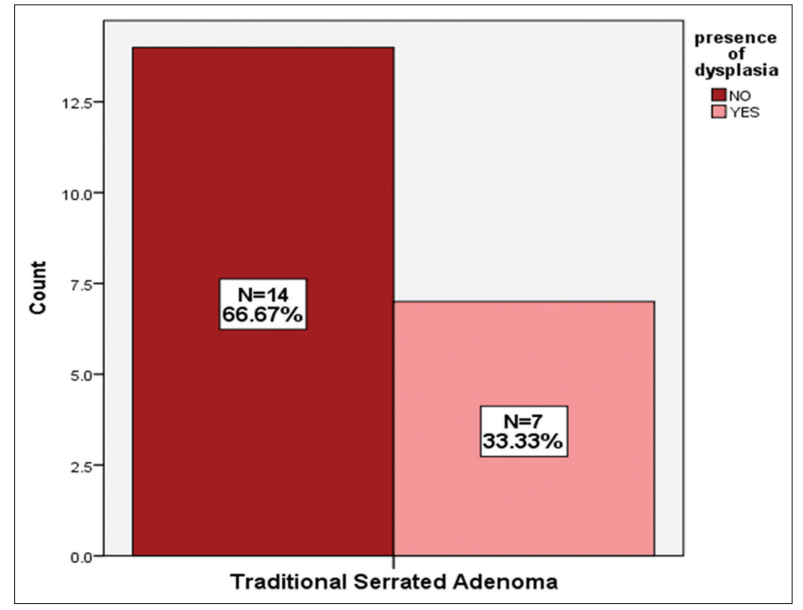

Figure 2: The prevalence of dysplasia in traditional serrated adenoma of this study

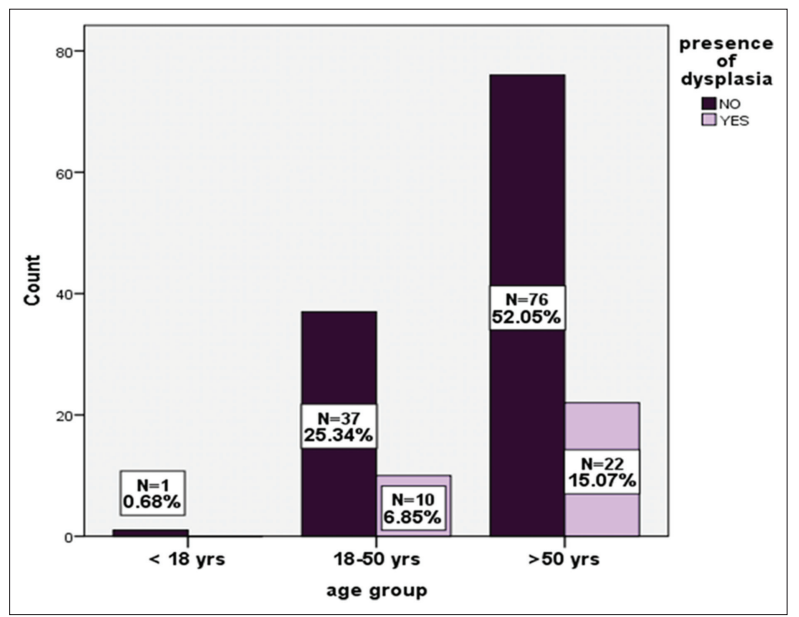

Figure 3: The prevalence of dysplasia amongst different age groups in this study 


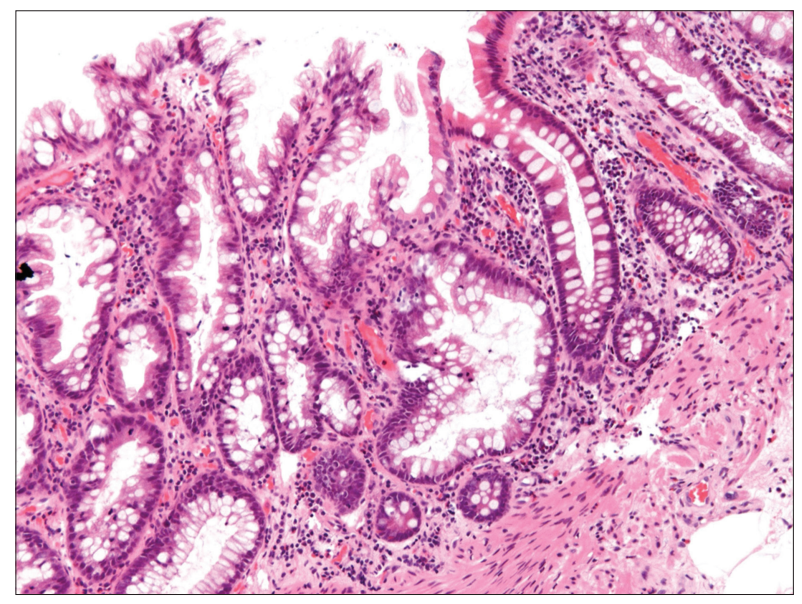

Figure 4: $\mathrm{H}$ and $\mathrm{E}$ stained side at $\times 20$ magnification showing surface serration, elongation of crypts and few boot-shaped glands. Mild dysplasia is noted in basal crypts

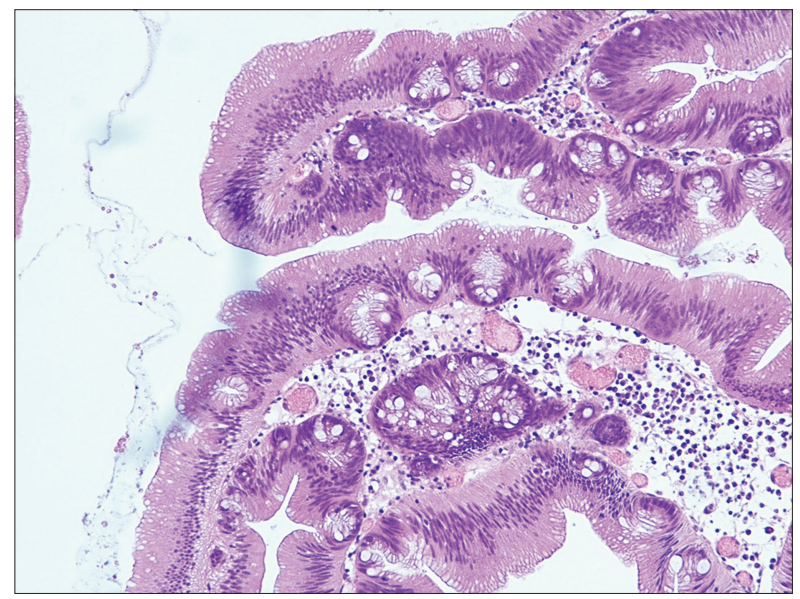

Figure 5: $\mathrm{H}$ and $\mathrm{E}$ stained section at $\times 20$ magnification showing villous architecture of surface with pencillate cells

the descending colon, sigmoid colon and rectum were regarded as distal colon.

Pathological parameters included type of polyp, and presence and degree of dysplasia. Polyps were divided into HP, SSA and TSA. Then, dysplastic changes were categorised into low-grade and high-grade dysplasia.

For data analysis, Statistical Package for the Social Sciences 23 version was used.

\section{Results}

This study included 146 biopsies over the duration of 3 years (2014-2016). 99 (67.8\%) were male and 47 (32.2\%) were female. Mean age was $55.68 \pm 13.74$ years (range: $17-83$ years). Majority were $>50$ years $98(67.1 \%)$, $47(32.2 \%)$ from 18 to 50 years and only one $(0.7 \%)$ patient was under 18 years. HPs were the most common type of serrated polyps 108 cases $(73.97 \%)$ followed by TSA 21 (14.38\%) and 17 (11.64\%) were reported as SSA. Hyperplastic polyps and TSAs were found commonly in rectum and descending colon, respectively, but SSA was common in ascending colon.

Overall dysplasia was seen in 32 of 146 cases $(21.9 \%)$ and 114 (78.1\%) were without dysplasia. Of these dysplastic, biopsies' only two cases (1.4\%) showed high-grade dysplasia and rest 30 (20.5\%) low-grade dysplasia.

Sessile serrated adenoma showed serrated crypts with boot shaped glands in the basal layers, with nuclear and architectural atypia [Figure 4]. Traditional serrated adenoma showed villous architecture with pencillate cells having eosinophilic cytoplasm [Figure 5]. According to the type of polyp, dysplasia was found in TSA $8 / 21$ cases $(46.7 \%)$ and SSA $9 / 17$ cases $(53.3 \%)$.

\section{Discussion}

To the best of our knowledge, this study is the first to demonstrate the prevalence of dysplasia in serrated/ hyperplastic lesions in Omani population.

In this study, we reviewed 146 colonic biopsies of serrated lesion over a period of 3 years (2014-2016).

Our study showed mean age of 55.68 with the range of $17-83$ years. On the other hand, another study showed the age mean of 61.6 years. Our study showed male predominance in the development of serrated polyps $(67.8 \%)$ which is consistent with other studies. ${ }^{[6,9]}$

According to our data, HPs have higher prevalence with the percentage of $73.97 \%$ compared to SSA/polyp and TSA which is consistent with reported literature. ${ }^{[10]} \mathrm{A}$ previous local study showed HPs to have a prevalence of $31.9 \%$ of all polyps. ${ }^{[9]}$

Regarding the dysplastic changes, our findings showed overall percentage of dysplasia to be $21.9 \%$; the result was similar to a study conducted in South Korea with percentage of $27.4 \% .{ }^{[11]}$ Of these, $46.7 \%$ of the TSA 
specimens and $53.3 \%$ of SSA/P showed dysplastic changes. The overall studies suggest that both SSA and TSA have malignant potential compared to HP. ${ }^{[12]}$

We found also that dysplasia detection was higher with older age group of $>50$ years and showed male predominance, this was consistent with global studies. ${ }^{[13]}$

Dysplasia was more common in distal colon compared to proximal with $P=0.005$. Although, other global studies showed higher prevalence of dysplasia in proximal colon. ${ }^{[13]}$

In summary, we report a high prevalence of HPs compared to SSA/polyp and TSA. Age and gender are important risk factors not only for developing serrated lesions but also increase the possibility of dysplastic changes. These are more common in SSA/polyp and traditional serrated. We also concluded that our data based on Omani population are comparable with international data.

\section{Conclusion}

Gastroenterologists should be aware of the emerging pattern of serrated lesions of colon and rectum that these are now common abnormality that they can face in their daily practice compared to past. This result could help guide gastroenterologists and endoscopy units in the on-going effort to improve detection and resection of precancerous serrated lesions. However, multicentre large sample-sized prospective studies are needed for further confirmation of our results. In addition, we require a better understanding of the molecular defects associated with serrated to optimally affect screening and prevention of all sporadic colorectal cancer.

\section{Conflict of Interest}

The authors declare that they have no conflict of interest.

\section{References}

1. Kumar VK, AbbasAC, Aster J. Robbins Basic Pathology. $9^{\text {th }}$ ed. Philadelphia, PA: Saunders; 2013.
2. Bosman FT, Carneiro F, Hruban RH, Theise ND. WHO classification of tumours of the digestive system. J Clin Ultrasound 2014;9:1-6.

3. Rex DK, Ahnen DJ, Baron JA, Batts KP, Burke CA, Burt RW, et al. Serrated lesions of the colorectum: Review and recommendations from an expert panel. Am J Gastroenterol 2012;107:1315-29.

4. Jouret-Mourin A, Geboes K. Serrated lesions of the colorectum, a new entity: What should a clinician/ endoscopist know about it? Acta Gastroenterol Belg 2012;75:197-202.

5. Burnett-Hartman AN, Passarelli MN, Adams SV, et al. Differences in epidemiologic risk factors for colorectal adenomas and serrated polyps by lesion severity and anatomical site. Am J Epidemiol 2013;177:625-37.

6. Haque T, Greene KG, Crockett SD. Serrated neoplasia of the colon: What do we really know? Curr Gastroenterol Rep 2014;16:380.

7. Bettington ML, Chetty R. Traditional serrated adenoma: An update. Hum Pathol 2015;46:933-8.

8. Patai AV, Molnár B, Tulassay Z, et al. Serrated pathway: Alternative route to colorectal cancer. World J Gastroenterol 2013;19:607-15.

9. Qureshi A, Shihi SA, Ali Z, et al. A retrospective study of clinico-pathological characteristics of colonic polyps in adults seen at a tertiary care centre. J Pak Med Assoc 2017;67:12-4.

10. Cao HL, Chen X1, Du SC, et al. Detection Rate, distribution, clinical and pathological features of colorectal serrated polyps. Chin Med J 2016;129:2427-33.

11. Haque TR, Bradshaw PT, Crockett SD. Risk factors for serrated polyps of the colorectum. Dig Dis Sci 2014;59: 2874-89.

12. Orlowska J. Serrated lesions and hyperplastic (serrated) polyposis relationship with colorectal cancer: Classification and surveillance recommendations. Gastrointest Endosc 2013;7:858-71.

13. Sweetser S, Smyrk TC, Sinicrope FA. Serrated colon polyps as precursors to colorectal cancer. Clin Gastroenterol Hepatol 2013;11:760-7.

\section{Authorship Contributions}

Concept and Design: AQ, NH, AS; Data Collection and interpretation: $\mathrm{NH}, \mathrm{AQ}$; Literature review and drafting: $\mathrm{NH}, \mathrm{AQ}$; Manuscript approval: AQ, NH, AS 